\title{
SEVERAL INTEGRAL INEQUALITIES ON TIME SCALES
}

\section{Li Yin, QIU-Ming LUO AND FENG QI}

Abstract. In this paper, we provide some new integral inequalities of Qi type on time scales by using elementary analytic methods.

Mathematics subject classification (2010): Primary 26E70; Secondary 26D15, 93C70. Keywords and phrases: Integral inequality, calculus of time scales, delta differentiability.

\section{REFERENCES}

[1] M. Akкоuchi, Some integral inequalities, Divulg. Mat. 11, 2 (2003), 121-125.

[2] M. Bohner AND A. Peterson, Dynamic Equations on Time Scales: An Introduction with Applications, Birkhäuser, Boston, 2001.

[3] K. Brahim, N. Bettaibi, And M. Sellemi, On some Feng Qi type q-integral inequalities, J. Inequal. Pure Appl. Math. 9, 2 (2008), Art. 43; Available online at http://www.emis.de/journals/JIPAM/article975.html.

[4] V. Krasniqi And A. Sh. Shabani, On some Feng Qi type h-integral inequalities, Int. J. Open Probl. Comput. Sci. Math. 2, 4 (2009), 516-521.

[5] Y. Miao And F. QI, Several q-integral inequalities, J. Math. Inequal. 3, 1 (2009), 115-121.

[6] F. QI, Several integral inequalities, J. Inequal. Pure Appl. Math. 1, 2 (2000), Art. 19; Available online at http://www.emis.de/journals/JIPAM/article113.html.

[7] L. Yin, On several new Qi's inequalities, Creat. Math. Inform. 20, 1 (2011), 90-95.

[8] L. YIN AND F. QI, Some integral inequalities on time scales, Available online at http://arxiv.org/abs/1105.1566. 